\title{
Morphological analysis of microglia in early postischemic period in the mouse local cerebral ischemia
}

\author{
Glyavina M.M. ${ }^{1,2}$, Loginov P.A. ${ }^{1}$, Dudenkova V.V. ${ }^{1,2}$, Shirokova O.M. ${ }^{1}$, Reunov D.G. ${ }^{1,2}$ \\ Karpova A.O. ${ }^{1,2}$, Prodanets N.N. ${ }^{1}$, Korobkov N.A. ${ }^{3}$, Zhuchenko M.A. ${ }^{4}$, Mukhina I.V. ${ }^{1,2}$ \\ ${ }^{1}$ Privolzhsky Research Medical University, Nizhny Novgorod, Russian Federation, mariyannov@gmail.com \\ ${ }^{2}$ Lobachevsky State University, Nizhny Novgorod, Russian Federation, \\ ${ }^{3}$ Saint Petersburg State University, Saint Petersburg, Russian Federation, \\ ${ }^{4}$ Pharmapark LLC, Moscow, Russian Federation.
}

Ischemic stroke is one of the most frequent causes of death and disability of patients. Microglia is a unique example of immunocompetent cell in central nervous system that is capable protecting the brain from ischemic injury. Microglia are constantly surveying their microenvironment. Under pathological conditions microglia rapidly change their morphology and adopt activation states in order adequately react to the activation-causing stimuli. However, now there is no understanding of the specific physiological role of each of the morphological microglia phenotypes $[1,3$, 6].

At present, drugs mainly aimed at minimizing the consequences of a stroke are intensely developed. One of the new lines in the development of neuroprotection methods in a stroke is to use a $\beta$ - common receptor subunit $(\beta-\mathrm{cR})$ with EPOR as a therapeutic target. One of microglia membrane's receptors is common receptor subunit $(\beta-\mathrm{cR})$, which in the presence of a ligand can form a dimeric complex with erythropoietin. Carbamylated darbopoietin (CdEPO) (PHARMAPARK LLC, Moscow) was used as an agonist for this receptor. When the receptor is connected to erythropoietin, this triggers two types of reactions, namely, a positive one, which activates the protective mechanisms of damaged neurons, and a negative one, which is caused by the increasing number of erythrocytes, resulting in reformation of a thrombus and a rise in blood pressure $[2,4]$. Therefore, Carbamylated darbopoietin (CdEPO) (Pharmapark LLC, Moscow) is able protect neurons from ischemic damage, ignoring hemopoiesis, which prevents the increase in hematocrit. Approximately $80 \%$ of the thrombotic or thromboembolic strokes occur in the middle cerebral artery. Thus, a model of transient occlusion of the middle cerebral artery (tOCMA) was used to study the stroke pathogenesis mechanism [5].

The somatosensory cortex and the hippocampus play an important role in learning and memory processes. Therefore, were investigated the morphological characteristics of microglia in these areas of brain.

Laser radiation is used to image the dynamic of changing microglia morphological phenotype. An objective of this work is to study the proportion of microglia phenotype and their morphological characteristic after of the middle cerebral artery occlusion (MCAO) and CdEPO treatment, because this is important for assessing the development of ischemic damage. The report presents the results of microglia phenotype distribution in the somatosensory cortex and hippocampus of the mouse brain in the early postischemic period in the MCAO model.

For this experiment, we use C57BL/6 mice. Surgery has been made using a binocular microscope and under isoflurane anesthesia. One-hour exposure was proceeded by a 7-0 microfilament from Doccol Corporation. The animal behavior was tested on the first day after the injection. Preservation of basic activity and memory was studied with Open Field LE800S test, Shuttle Box LE916 (PanLab/Harvard Apparatus), "novel object recognition" test. CdEPO was injected after intravenously in 1, 3, 6, 12, 24, 48 hours after model of transient occlusion of the middle cerebral artery (tMCAO) (one-hour exposure) at $50 \mathrm{mkg} / \mathrm{kg}$ dose. Behavior was studied on the 4-th postoperative day. Brain is fixed in 4\% paraformaldehyde (PFA). Microglia was marked with primary Iba-1 antibodies (Rabbit polyclonal) and secondary antibody with fluorescent agant AlexaFluor 555. Visualization was carried out by a ZEISS LSM 880 laser scanning confocal fluorescents microscope with an 40x immersion objective and a yellow-green spectrum filter. Paraffin coronal sections of the brain $(5 \mu \mathrm{m}$ thick) were studied. The images were processed using ImageJ and Python 3 software.

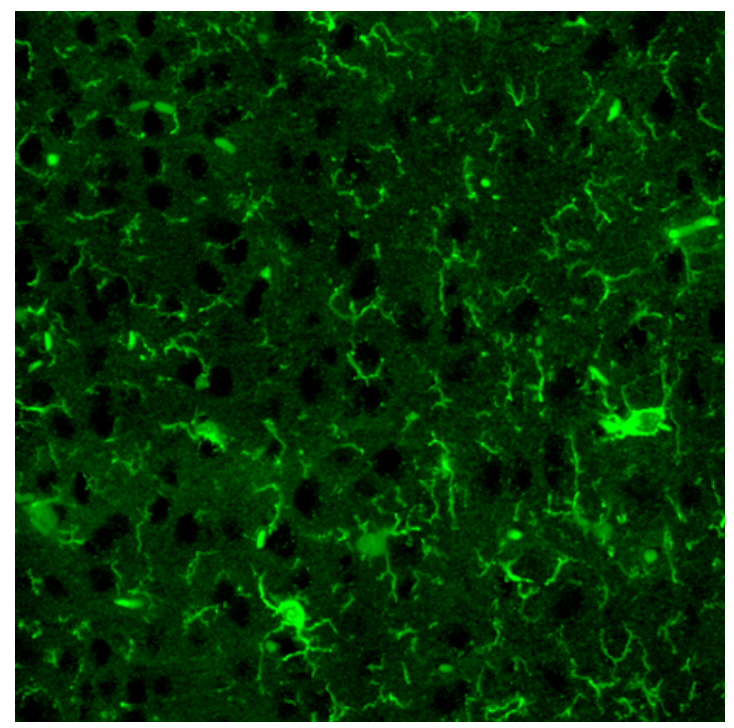

Fig. 1. Example of the processed image. The region of somatosensory cortex in the mouse brain (intact group). Green color corresponded to microglia (iba-1)

The morphological characteristics of microglia in the hippocampus is researched in the hemisphere with 
an ischemic injury. The number of microglial cells in the hippocampus were decreased in control group. In the group with CdEPO injection, the number of microglia cells had not changed. The area of microglial cells decreased in the control group and rise in the group with injection of CdEPO on the 4th day after the MCAO in the hippocampus. On the 4th day after $\mathrm{MCAO}$, there was a decline in the number of ramified microglia in the control group and in group with injection of CdEPO. However, generation of active microglia was rise in in the control group and in group with injection of CdEPO. Moreover, generation of amoeboid microglia was doubled in control group and had not changed in group with injection of CdEPO.

Microglia in the somatosensory cortex were studied in the hemisphere with an ischemic injury and in the contralateral zone. The number of microglial cells in the somatosensory cortex in the zone with ischemic damage decreased in control group. In the group with CdEPO injection there is an opposite situation: number of microglial cells approximated the intact values. The area of microglial cells decreased on the 4th day after the MCAO in the control group and in the group with injection of CdEPO. On the 4th day after $\mathrm{MCAO}$, there was a decline in the number of ramified microglia in the control group as well as in generation of active and amoeboid microglia in both hemispheres. In the group with CdEPO injection, the number of resting microglia had not changed but the amount of activated microglia increased.

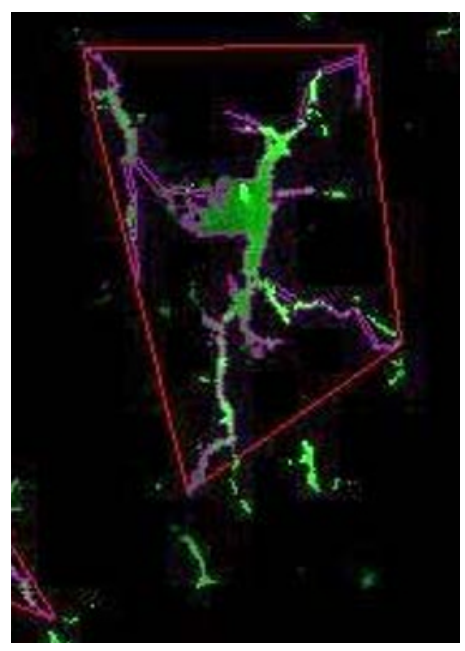

Fig. 2. Dendritic tree area (inside the solid - red in color online - line area). Area of contact microglia cell and microenvironment.

Change in microglia phenotypes and their morphology characteristic proportion in the group with CdEPO injection correlated with improved functional state of the animals after one-hour exposure: their motor activity restored, stress level was decreased and their memory was preserved.

The program of evaluating the morphological characteristics of microglia will help to understand the contribution of various microglia phenotypes to development of the ischemic damage and restoring the organism in the post-ischemic period. The ongoing research on regulation of microglia phenotypes pro- portion with CdEPO allows considering it as a promising neuroprotector giving a positive impact on the organism recovery in early post-ischemic period.

The reported study was funded by RFBR according to the research project № 18-34-00877

\section{References}

1. Bachstetter $A D$ et all, Disease-related microglia heterogeneity in the hippocampus of Alzheimer's disease, dementia with Lewy bodies, and hippocampal sclerosis of aging// Acta Neuropathol Commun., 2015 No. 23. P.3- 32.

2. James M., Ian G. Young, IL-3, IL-5, and GM-CSF signaling: crystal structure of the human beta-common receptor // Vitam Horm. 2006. No.74. P.1-30

3. Karperien A. $L$ et all., Box-counting analysis of microglia form in analysis of microglia form in schizophrenia, alzheimer's disease and affective disorder// Fractals, 2008. V. 16. No. 2. P. 103-107.

4. Nataša Debeljak et all, Erythropoietin and cancer: the unintended consequences of anemia correction // Front. Immunol., 2014. No. 5. P.563

5. Rousselet1 E., Kriz J., Seidah N.G., Mouse Model of Intraluminal MCAO: Cerebral Infarct Evaluation by Cresyl Violet Staining // J. Vis. Exp., 2012. No.69. pii: 4038.

6. Youngjeon Lee et all, Therapeutically Targeting Neuroinflammation and Microglia after Acute Ischemic Stroke// BioMed Research International, 2014. Article ID 297241, P.1-9. 